OPEN ACCESS

Edited by:

Yu Wang,

Chinese Academy of Sciences, China

Reviewed by:

Qiang Fei,

Xi'an Jiaotong University, China

Marina G. Kalyuzhanaya,

San Diego State University,

United States

Muhammad Farhan UI Haque, University of the Punjab, Pakistan

*Correspondence:

Toshiaki Kamachi

tkamachi@bio.titech.ac.jp

Specialty section: This article was submitted to Microbiotechnology,

a section of the journal

Frontiers in Microbiology

Received: 08 December 2020 Accepted: 28 February 2021

Published: 22 March 2021

Citation:

Ito $\mathrm{H}$, Yoshimori K, Ishikawa $\mathrm{M}$, Hori K and Kamachi T (2021)

Switching Between Methanol Accumulation and Cell Growth by

Expression Control of Methanol

Dehydrogenase in Methylosinus trichosporium OB3b Mutant.

Front. Microbiol. 12:639266.

doi: 10.3389/fmicb.2021.639266

\section{Switching Between Methanol Accumulation and Cell Growth by Expression Control of Methanol Dehydrogenase in Methylosinus trichosporium OB3b Mutant}

\author{
Hidehiro Ito ${ }^{1,2}$, Kosei Yoshimori ${ }^{1}$, Masahito Ishikawa ${ }^{2}$, Katsutoshi Hori ${ }^{2}$ and \\ Toshiaki Kamachi ${ }^{1 *}$ \\ ${ }^{1}$ Department of Life Science and Technology, Tokyo Institute of Technology, Tokyo, Japan, ${ }^{2}$ Department of Biomolecular \\ Engineering, Nagoya University, Nagoya, Japan
}

Methanotrophs have been used to convert methane to methanol at ambient temperature and pressure. In order to accumulate methanol using methanotrophs, methanol dehydrogenase $(\mathrm{MDH})$ must be downregulated as it consumes methanol. Here, we describe a methanol production system wherein MDH expression is controlled by using methanotroph mutants. We used the MxaF knockout mutant of Methylosinus trichosporium OB3b. It could only grow with MDH (XoxF) which has a cerium ion in its active site and is only expressed by bacteria in media containing cerium ions. In the presence of $0 \mu \mathrm{M}$ copper ion and $25 \mu \mathrm{M}$ cerium ion, the mutant grew normally. Under conditions conducive to methanol production (10 $\mu \mathrm{M}$ copper ion and $0 \mu \mathrm{M}$ cerium ion), cell growth was inhibited and methanol accumulated $\left(2.6 \mu \mathrm{mol} \cdot \mathrm{mg}^{-1} \mathrm{dry}\right.$ cell weight $\left.\cdot \mathrm{h}^{-1}\right)$. The conversion efficiency of the accumulated methanol to the total amount of methane added to the reaction system was $\sim 0.3 \%$. The aforementioned conditions were repeatedly alternated by modulating the metal ion composition of the bacterial growth medium.

Keywords: methanotroph, methane, methanol, methane monooxygenase, methanol dehydrogenase

\section{INTRODUCTION}

Methane is a principal component of natural and shale gases. As its global reserves are vast, it is regarded as a next-generation carbon feedstock. Biogas produced by microbial biomass digestion under anaerobic conditions is composed mainly of methane. Microbial methane fermentation can use carbon dioxide as a carbon source. Therefore, methane is expected to be a valuable industrial fuel source as it is renewable and the technologies associated with its collection, production, and consumption are sustainable. Nevertheless, its global warming potential is $25 \times$ higher than that of carbon dioxide [U.S. Environmental Protection Agency (EPA), 2019]. Hence, it is a prime mitigation target in greenhouse gas reduction (Shindell et al., 2012). A major source of atmospheric methane is the anaerobic decomposition of biomass from anthropogenic industrial waste production and agricultural activity (Conrad, 2009). However, the methane produced by archaea is low concentration and the collectable gas contains 
impurities such as carbon dioxide. Thus, it can only be used as fuel without any purification. In contrast, methanol is readily available as a carbon feedstock for further chemical conversion and is easy to store and transport because it is a liquid. Therefore, the efficient conversion of methane to methanol may help increase the availability of heretofore underutilized methane resources.

As methanotrophs are powerful oxidizers, their exploitation in the industrial oxidation of methane to methanol has been investigated. Aerobic methanotrophs oxidize methane to methanol via methane monooxygenase (MMO) and $\mathrm{NADH}$ at ambient temperature and pressure. Thence, the methanol is oxidized to formaldehyde by methanol dehydrogenase (MDH). The formaldehyde may either be assimilated into biomass or oxidized to formate by formaldehyde dehydrogenase (FADH). The formate is then converted to carbon dioxide via formate dehydrogenase $(\mathrm{FDH})$, the $\mathrm{NADH}$ is regenerated for $\mathrm{MMO}$, and the cycle is completed. The simplest and most cost-effective approach to the foregoing process is the use of whole-cell methanotroph cultures (Bjorck et al., 2018). Whole cells undergo autopoiesis by biosynthesizing enzymes and replicating themselves. Moreover, the methanol biosynthesized by whole cells can be easily separated. In methanol biosynthesis via MMO and methane oxidation, reducing equivalents are required. Whole-cell methanotrophs can supply the reducing equivalent in the form of NADH. A major problem with the application of whole-cell methanotrophs for methanol production is that the microorganisms continue to oxidize the methanol to formaldehyde and thence to formate. Therefore, methanol oxidation must be interrupted so that the methanol can accumulate. The irreversible MDH inhibitor cyclopropanol binds pyrroloquinoline quinone (PQQ) and deactivates MDH (Frank et al., 1989; Shimoda and Okura, 1990; Takeguchi et al., 1997; Furuto et al., 1999). High phosphate or sodium chloride concentrations in the medium also promote methanol accumulation (Mehta et al., 1987; Cox et al., 1992; Dales and Anthony, 1995; Lee et al., 2004; Duan et al., 2011; Han et al., 2013). However, complete MDH inhibition arrests cell growth and the loss of reducing power it causes impedes the natural biochemical pathways of the cell. For efficient methanol production by $\mathrm{MDH}$ inhibition, external electron donors such as formate must be added to regenerate NADH (Mehta et al., 1991; Furuto et al., 1999; Lee et al., 2004; Kim et al., 2010; Duan et al., 2011; Pen et al., 2014, 2016). Even when these are supplied, however, the methanotrophs cannot be reused as their MMO activity is destabilized. Hence, the strategic design of methanol production and cell growth with enzyme recovery and reducing equivalents is necessary for continuous methanol production with whole-cell methanotrophs. In this process, it is important to control $\mathrm{MDH}$ activity in the bacteria. Lowering $\mathrm{MDH}$ activity causes methanol to accumulate, restores $\mathrm{MDH}$ activity, and provides intracellular reducing power. However, it is difficult to modulate intracellular $\mathrm{MDH}$ activity via conventional inhibitor-based methods.

To solve this problem, we examined gene regulation systems in methanotrophs in response to certain metal ions. The canonical "copper switch" controls the expression of two types of MMO (Yoon et al., 2010). Particulate MMO (pMMO) is localized to the intracytoplasmic membranes and its expression and activity increase with copper ion availability. In contrast, soluble MMO (sMMO) occurs in the cytoplasm and is only expressed in the absence of copper ions. $\mathrm{MxaF}$ and XoxF are forms of the main component of PQQ-dependent $\mathrm{MDH}$ and are found in certain methanotrophs such as Methylosinus trichosporium OB3b. MxaF has a calcium ion in its active site (Williams et al., 2005) whereas the active site of XoxF has a rare earth element such as a cerium, lanthanum, or praseodymium ion (Hibi et al., 2011; Nakagawa et al., 2012; Keltjens et al., 2014; Pol et al., 2014). Rare earth elements are gene-regulating factors and control MxaF and XoxF expression. Farhan Ul Haque et al. (2015) first reported that $m x a F$ and $x o x F$ expression in $M$. trichosporium OB3b substantially decreased and increased, respectively, with increasing cerium ion concentration. This mechanism is known as the "lanthanide switch" (Vu et al., 2016; Groom et al., 2019). Therefore, this lanthanide switch could effectively control MDH activity in whole-cell methanotrophs.

Here, we used the MxaF knockout mutant of M. trichosporium $\mathrm{OB} 3 \mathrm{~b}$ to test the aforementioned strategic methanol production and cell growth design (Figure 1). This mutant was constructed by referring to the methods of previous studies (Semrau et al., 2013; Farhan Ul Haque et al., 2016). This mutant can only use XoxF MDH for methanol oxidation. Hence, XoxF MDH downregulation is expected to result in methanol generation in the absence of cerium. This mutant can again resume growth back by re-expression of XoxF MDH in the presence of cerium. In addition, $\mathrm{NADH}$ recycling and/or MMO refreshing through the methanol metabolic pathway restoration may be achieved. Using the lanthanide switch over a long methanol production period without the addition of an external electron source. In this study, we reported switching of methanol production and cell growth in this mutant by modulating the copper and cerium ion content in the growth medium.

\section{MATERIALS AND METHODS}

\section{Microbial Growth Conditions}

Nitrate mineral salt (NMS) medium (Whittenbury et al., 1970) was used to grow wild type $M$. trichosporium OB3b and the $m x a F$ knockout mutant $(\Delta m x a F$, Table 1). Methane was the sole carbon and energy source in the growth substrate. Cultures were supplemented with copper ion $\left(\right.$ as $\mathrm{CuSO}_{4}$ ) and/or cerium ion $\left(\right.$ as $\mathrm{CeCl}_{3}$ ) from stock solutions prepared in ultrapure water. Copper or cerium ion stock solutions were sterilized by filtration through polyvinylidene fluoride (PDVF) membranes with $0.22-\mu \mathrm{m}$ pore size. Methylosinus trichosporium OB3b wild type and $\triangle m x a F$ cultures were grown at $30^{\circ} \mathrm{C}$ with constant shaking at $130 \mathrm{rpm}$ and under a 1:4 (v/v) methane/air mixture in $30 \mathrm{ml} \mathrm{NMS}$ in 200-ml Erlenmeyer flasks fitted with baffles. All reagents were obtained from commercial suppliers and were of the highest available purity.

A starter culture grown in NMS with $25 \mu \mathrm{M}$ cerium ion was used as an inoculum to examine the growth of the OB3b $\triangle m x a F$ mutant in the presence of copper and cerium ions. 

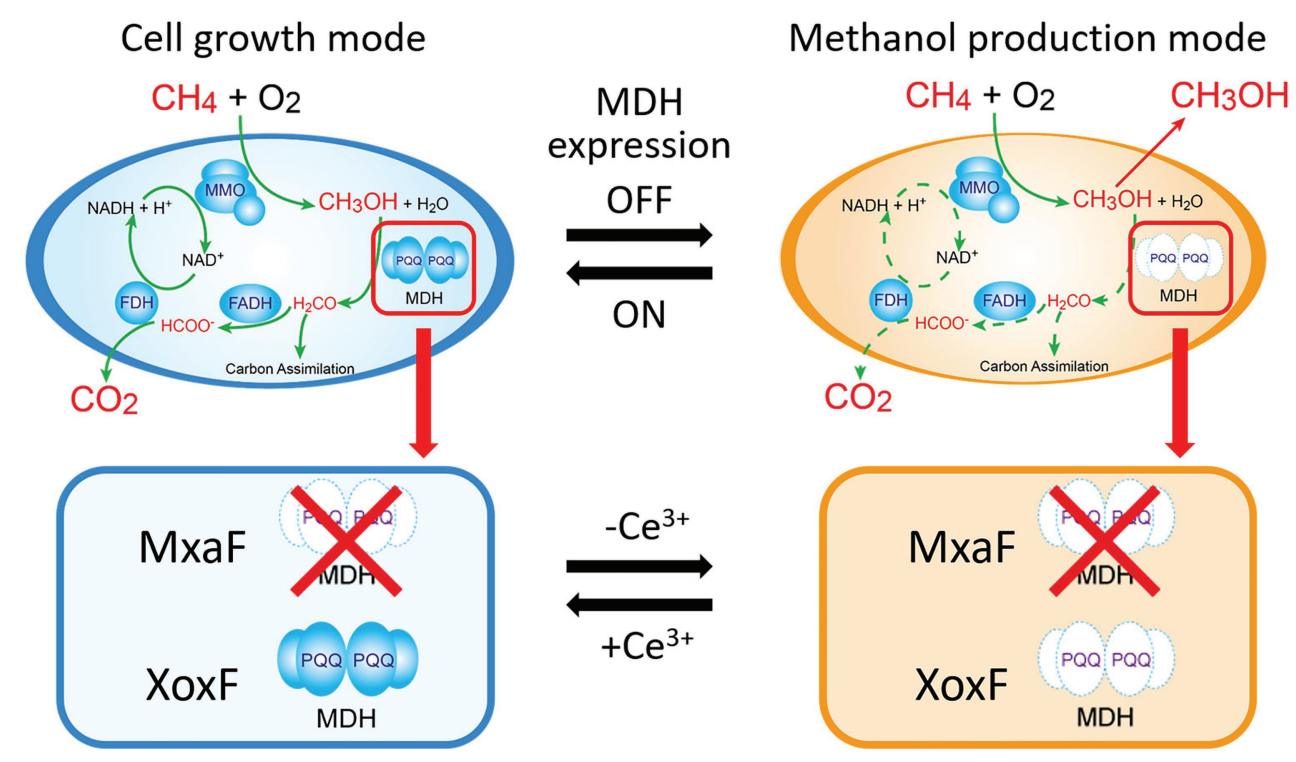

FIGURE 1 | Cell growth and methanol production induced in methanotrophs by switching methanol dehydrogenase gene expression. Solid green line in the cell growth mode means the metabolic pathway of methane in the presence of cerium ions. Dotted green line in the methanol production mode means that the pathway is blocked in the absence of cerium ions. MMO: methane monooxygenase; MDH: methanol dehydrogenase; FADH: formaldehyde dehydrogenase; FDH: formate dehydrogenase; $\mathrm{PQQ}$ : pyrroloquinoline quinone.

At the late exponential phase, the cultures were harvested by centrifugation at $3,000 \times \mathrm{g}$ and $4^{\circ} \mathrm{C}$ for $10 \mathrm{~min}$. The cell pellets were washed twice in NMS free of copper and cerium. After each washing, the pellets were resuspended in an equal volume of NMS free of copper and cerium and centrifuged at $3,000 \times \mathrm{g}$ and $4^{\circ} \mathrm{C}$ for $10 \mathrm{~min}$. After the second washing, the cell pellets were resuspended in NMS and inoculated into new cultures in 50-ml serum bottles each containing $10 \mathrm{ml}$ NMS. The initial target $\mathrm{OD}_{540}$ was 0.1 . Four different culture conditions were considered and cell growth and methanol production were measured under various metal ions concentrations. The bottles were sealed with rubber stoppers and aluminum caps and the headspaces were filled with a 1:4 (v/v) methane/air mixture. The serum bottles were incubated at $30^{\circ} \mathrm{C}$ and $130 \mathrm{rpm}$ in a shaking incubator (BR-43FL-MR; TAITEC, Saitama, Japan). At the stationary phase, the cultures were harvested by centrifugation at $3,000 \times \mathrm{g}$ and $4^{\circ} \mathrm{C}$ for $10 \mathrm{~min}$ and washed twice under the aforementioned conditions. In one experiment, cell pellets were transferred to $50-\mathrm{ml}$ serum bottles each containing $10 \mathrm{ml}$ identical fresh medium and the initial target $\mathrm{OD}_{540}$ was 0.1 (transfer A). In another experiment, cell pellets were transferred to $50-\mathrm{ml}$ serum bottles each containing $10 \mathrm{ml}$ identical fresh medium (transfer B). To toggle between cell growth and methanol production, the initial medium $\mathrm{OD}_{540}$ was adjusted to 0.1 after inoculation.

Escherichia coli were grown in Luria-Bertani (LB) medium. Gentamicin $\left(10 \mu \mathrm{g} \mathrm{ml} \mathrm{m}^{-1}\right)$ or kanamycin $\left(20 \mu \mathrm{g} \mathrm{ml}^{-1}\right)$ was used to conserve E. coli containing mutant construct plasmids and $M$. trichosporium OB3b mutant cultures after the first homologous recombination.

\section{Construction of the Methylosinus trichosporium OB3b $\Delta m \times a F$ Mutant}

The double homologous recombination method was used to construct the OB3b $\triangle m x a F$ mutant by referring to the methods of previous studies (Supplementary Figure S1; Semrau et al., 2013; Farhan Ul Haque et al., 2016). Briefly, pJQ200SK and pk18mobsacB plasmids were amplified in E. coli Turbo by the Inoue method (Sambrook and Russell, 2006). Two DNA fragments $\sim 1 \mathrm{~kb}$ distant from the $5^{\prime}$ to $3^{\prime}$ ends (arms A and B, respectively) of $m x a F$ in $M$. trichosporium OB3b were amplified by PCR. Specific primers (Table 2) were designed to amplify arms A and $\mathrm{B}$, introduce the Bam $\mathrm{HI}$ restriction site sequences, and facilitate directional cloning into the pJQ200SK and pk18mobsacB suicide vectors. The PCR products of arms A and B were gel-purified with a QIAquick gel extraction kit (Qiagen, Valencia, CA, United States) following the manufacturer's instructions. The pJQ200SK plasmid was digested with BamHI and gel-purified with a QIAquick gel extraction kit. The PCR product and linearized pJQ200SK were ligated with an In-Fusion cloning kit (TaKaRa Bio Inc., Kusatsu, Shiga, Japan) following the manufacturer's instructions. Then $m x a F$ in the pJQ200SK UpMxaDw plasmid was removed with PrimeStar Max DNA polymerase (TaKaRa Bio Inc., Kusatsu, Shiga, Japan) following the deletion protocol. The resulting plasmid was named pJQ200SK_ $\mathrm{UpDw}$ and digested with $\mathrm{BamHI}$. The connected regions upstream and downstream from mxaF (UpDw arm) were gel-purified with a QIAquick gel extraction kit. The pk18mobsacB plasmid was digested with BamHI and gel-purified with a QIAquick gel extraction kit. The UpDw arm and linearized pk18mobsacB were ligated with a ligation-convenience kit (NIPPON GENE, Toyama, Japan). The resulting plasmid was the final construct. 
TABLE 1 | Bacterial strains and plasmids used in this study.

\begin{tabular}{|c|c|c|}
\hline Strain or plasmid & Description & Reference or source \\
\hline \multicolumn{3}{|c|}{ Methylosinus trichosporium ОВ3b } \\
\hline Wild type & Wild type strain & ATCC35070 \\
\hline SC-SacB & $\begin{array}{l}\text { Single-crossover mutant } \\
\text { constructed by integration of } \\
\text { pK18mobsacB_UpDw in } \\
\text { flanking region of } m \times a F \text { of } \\
M \text {. trichosporium OB3b }\end{array}$ & In this work \\
\hline OB3b $\Delta m x a F$ & $\begin{array}{l}\text { Unmarked mutant of } m \times a F \\
\text { (METTRDRAFT_RS0210410) }\end{array}$ & In this work \\
\hline \multicolumn{3}{|c|}{ Escherichia coli } \\
\hline Turbo & $\begin{array}{l}\text { F proA+B+laclq } \triangle \text { lacZM15/ } \\
\text { fhuA2 } \triangle \text { (lac-proAB) glnV galK16 } \\
\text { galE15 R(zgb-210::Tn10) Tets } \\
\text { endA1 thi-1 } \triangle(\text { hsdS-mcrB)5 }\end{array}$ & NEB product info. \\
\hline S17-1 & $\begin{array}{l}\text { recA1 thi pro hsdR-RP4- } \\
\text { 2Tc::Mu Km:Tn7 }\end{array}$ & Simon, 1984 \\
\hline \multicolumn{3}{|l|}{ Plasmids } \\
\hline pJQ200SK & $\begin{array}{l}\text { Suicide vector, P15A, traJ, oriT, } \\
\text { sacB, Gm }\end{array}$ & Quandt and Hynes, 1993 \\
\hline $\begin{array}{l}\text { pJQ200SK_ } \\
\text { UpMxaDw }\end{array}$ & $\begin{array}{l}\text { DNA fragment containing from } \\
\text { upstream to downstream } \\
\text { regions of } m x a F \text { ligated into } \\
\text { BamHI site of pJQ200SK }\end{array}$ & In this work \\
\hline pJQ200SK_UpDw & $\begin{array}{l}\text { DNA fragment containing } \\
\text { upstream and downstream } \\
\text { regions of } m x a F \text { ligated into } \\
\text { BamHI site of pJQ200sk }\end{array}$ & In this work \\
\hline pK18mobsacB & $\begin{array}{l}\text { Suicide vector, oriT, } \mathrm{Km} \text { ', lacZ, } \\
\text { sacB }\end{array}$ & Schäfer et al., 1994 \\
\hline $\begin{array}{l}\text { pK18mobsacB_- } \\
\text { UpDw }\end{array}$ & $\begin{array}{l}\text { DNA fragment containing } \\
\text { upstream and downstream } \\
\text { regions of } m \times a F \text { ligated into } \\
\text { BamHI site of pK18mobsacB }\end{array}$ & In this work \\
\hline
\end{tabular}

$\mathrm{Km}$, kanamycin resistance; $\mathrm{Gm}$ ', gentamicin resistance.

It was named pK18mobsacB_UpDw, transferred to chemically competent E. coli S17.1 $\lambda$ pir (Simon, 1984) by the heat shock method, and transferred to $M$. trichosporium OB3b via conjugation between the donor (E. coli S17.1 $\lambda$ pir containing the pK18mobsacB_ $\mathrm{UpDw}$ construct) and the recipient (M. trichosporium OB3b) strains according to previously described methods (Martin and Murrell, 1995; Farhan Ul Haque et al., 2016). The mixture (1:5 donor:recipient ratio at $\mathrm{OD}_{540}$ ) was passed through a $0.2-\mu \mathrm{m}$ nitrocellulose filter (ADVANTEC Co. Ltd., Tokyo, Japan). The obtained filter was then incubated at $30^{\circ} \mathrm{C}$ on NMS agar containing $0.02 \%(\mathrm{w} / \mathrm{v})$ proteose peptone under a 50:50 (v/v) methane/air atmosphere for $24 \mathrm{~h}$. The cells were removed by vortexing the filter in $1 \mathrm{ml}$ NMS medium. The suspension was spread onto NMS agar plates containing $10 \mu \mathrm{g} \mathrm{ml}^{-1}$ kanamycin to select for $M$. trichosporium $\mathrm{OB} 3 \mathrm{~b}$ harboring the kanamycin resistance cassette and other pK18mobsacB_UpDw regions and $10 \mu \mathrm{g} \mathrm{ml}^{-1}$ nalidixic acid to prevent $E$. coli growth. The NMS agar plates were incubated at $30^{\circ} \mathrm{C}$ under a 50:50 (v/v) methane/air atmosphere for 2-3 weeks. The M. trichosporium OB3b colonies (first homologous recombinants; $M$. trichosporium OB3b SC-SacB) were suspended in NMS and inoculated onto NMS agar plates containing $0.5 \%(\mathrm{w} / \mathrm{v})$ sucrose to select for $M$. trichosporium $\mathrm{OB} 3 \mathrm{~b}$ without the $s a c B$ regions and $25 \mu \mathrm{M}$ cerium to express
TABLE 2 | Primers used in this study.

\begin{tabular}{ll}
\hline Name & Sequence 5'>3' \\
\hline Nup-F (armA) & CAGCCCGGGGGATCCAATGTGGGTTCGGTC \\
Ndown-R (armB) & AGAACTAGTGGATCCTCAATGAGCGAGCG \\
DLmxaF-F & TCGTCGATAACTGACCCTTCGCCGC \\
DLmxaF-R & GGTCAGTTATCGACGAGTCCTCCTGC \\
mxaF_upst-F1 (P1) & CACCATTCGCCTATCGTCTCAATC \\
mxaF_dwst-R1 (P2) & CCGGTGACCGTGTTGTGAAAG \\
mxaF-F2 (P3) & TACCGAGCTCGAATTCAGGAGGACTCGTCGATGAG \\
mxaF_R2 (P4) & GAAG \\
mxaF_mid-F & ACGGCCAGTGAATTCTCAGTTCGCCTTGTACTCGCC \\
mxaF_mid-R & TTCGTCAGAGCCTCGAGCTTGTCCTC \\
mxaF_upst-F2 & GGCGAGAATATTGGCGTAGGCCGGATAG \\
mxaF_dwst-R2 & TGGGGTCATGCTTGGGATCGTATTCGAG \\
pK18mobsacB_seq-F & AAGCCCACTGCAAGCTACCTG \\
pK18mobsacB_seq-R & ACCTACACCGAACTGAGATACCTAC \\
&
\end{tabular}

Underlined sequences represent different restriction sites.

XoxF instead of MxaF. The NMS agar plates were incubated at $30^{\circ} \mathrm{C}$ under a 50:50 (v/v) methane/air atmosphere for 10 days. The $M$. trichosporium OB3b colonies (second homologous recombinants; $M$. trichosporium OB3b $\Delta m x a F$ mutant) obtained were suspended in NMS containing $25 \mu \mathrm{M}$ cerium. PCR and sequencing analysis confirmed success of the double homologous recombinants and crossover mutant genotypes.

\section{Methanol Concentration}

Methanol concentrations were measured by a gas chromatograph (GC-2014; Shimadzu Corp., Kyoto, Japan) fitted with a flame ionization detector (FID) and a packed glass column $(2 \mathrm{~m} \times$ $3 \mathrm{~mm}$ i.d.; GL Science, Tokyo, Japan) containing sorbitol 25\%-gasport B (60/80 mesh; GL Science, Tokyo, Japan). Nitrogen was the carrier gas and the flow rate was $40 \mathrm{ml} / \mathrm{min}$. The operating temperatures were injection, $150^{\circ} \mathrm{C}$; column, $100^{\circ} \mathrm{C}$; and detector, $150^{\circ} \mathrm{C}$. Five microliters sample solution was injected for each measurement.

\section{Immunodetection}

Anti-XoxF and anti-MxaF antisera were generated against synthesized peptides corresponding to residues 339-351 of MxaF and residues 374-387 of XoxF1, respectively. Whole-cell lysates of $M$. trichosporium OB3b and OB3b $\Delta m x a F$ mutant were separated on $10 \%(\mathrm{w} / \mathrm{v})$ acrylamide gel and transferred to a PVDF membrane following general protocols. The Precision Plus Protein ${ }^{\mathrm{TM}}$ Dual Color Standards (Bio-Rad Laboratories, Hercules, CA, United States) were used for the molecular weight marker. The blotted membrane was blocked with 5\% (w/v) skim milk at room temperature for $1 \mathrm{~h}$ and treated at room temperature for $1 \mathrm{~h}$ with anti-MxaF or anti-XoxF1 antisera at 1:2,000 dilution in Tris-buffered saline (TBS) containing 0.05\% (v/v) Tween 20 (TBS-T; Calbiochem, San Diego, CA, United States). Membrane-bound MxaF and XoxF1 were detected with horseradish peroxidase-conjugated anti-rabbit IgG antibody (GE Healthcare, Little Chalfont, United Kingdom) at 1:10,000 dilution in TBS-T and visualized using EzWestLumi Plus (ATTO Corp., Tokyo, Japan) according to the manufacturer's instructions. 
A

M. trichosporium OB3b WT

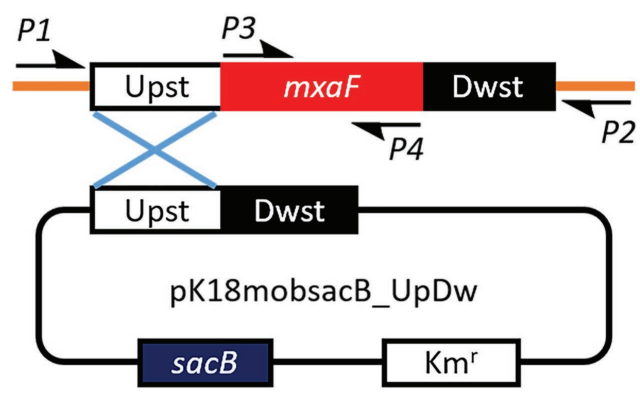

1st recombination
with $\mathrm{Km}$

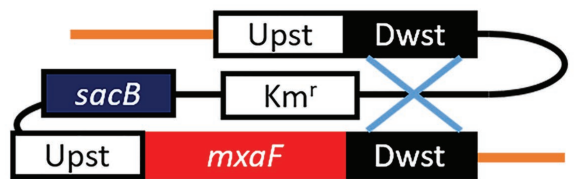

2nd recombination
with sucrose, $\mathrm{Ce}^{3+}$

M. trichosporium OB3b $\Delta m x a F$
B

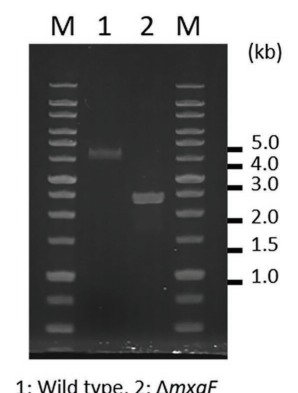

C

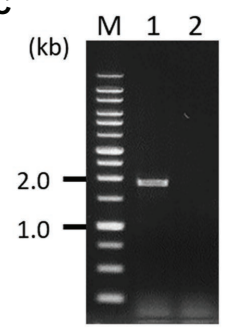

D

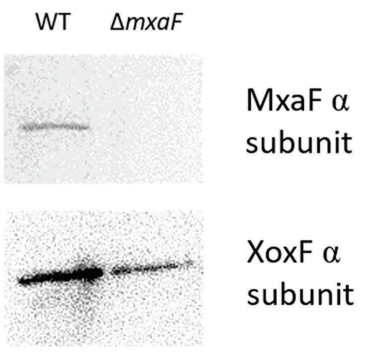

FIGURE 2 | Plasmid-based counterselection. (A). Suicide plasmids used Kmr (kanamicine resistance gene) and sacB as counterselectable markers. Half arrows indicate primers (P1, mxaF_upst-F1; P2, mxaF_dwst-R1; P3, mxaF-F; P4, mxaF-R). Primer nucleotide sequences are shown in Table 2. WT, wild type; $\Delta m x a F$, MxaF knockout mutant. PCR confirmation of $m x a F$ disruption using primers P1 and P2 (B), P3 and P4 (C). From genome sequence information for

M. trichosporium OB3b, lengths of PCR amplicons using primers P1 and P2 from wild type and $\triangle m x a F$ mutant are expected to be 4.3 and 2.4 kbp, respectively

(B). PCR amplicons using primers P3 and P4 are expected to be $1.9 \mathrm{kbp}$ from wild type only (C). MxaF and XoxF immunodetection using specific antisera (D).

\section{RESULTS}

\section{Construction of Methylosinus trichosporium OB3b $\Delta m \times a F$ Mutant}

We constructed a mutant of $\triangle m x a F$ in $M$. trichosporium OB3b using counterselection and double homologous recombination methods with $s a c B$ as a counterselectable marker (Figure 2A). We prepared the plasmids pJQ200SK_ UpDw and pK18mobsacB_UpDw (Supplementary Figure S1). However, construction of the mutant succeeded only when pK18mobsacB_UpDw was used. Plasmid integration into the target site was confirmed by PCR with the primer sets A (mxaF_upst-F2 and mxaF_mid-R) and B (mxaF_dwst-R2 and mxaF_mid-F) on a single-crossover mutant grown on NMS with kanamycin (Supplementary Figure S2). The single-crossover mutant was plated on NMS containing sucrose and cerium for counterselection. We applied PCR to the surviving colonies and selected double-crossover mutants using primers $\mathrm{P} 1$ and P2. The PCR amplicons from the $\triangle m x a F$ mutants were shorter $(2.4 \mathrm{kbp})$ than those from the wild type $(4.3 \mathrm{kbp}$; Figure $2 \mathrm{~B})$. The PCR product was confirmed by sequencing. No PCR product from the $\Delta m x a F$ mutants was detected using primers P3 and P4 (Figure 2C). Hence, $m x a F$ and the region derived from the integrated plasmid were successfully deleted. It was established that the mutant was resistant to sucrose but sensitive to kanamycin. Immunodetection with anti-MxaF and anti-XoxF antisera demonstrated the absence of $\mathrm{MxaF}$ expression and the presence of XoxF expression in the $\triangle m x a F$ mutant (Figure 2D). Thus, the targeted mutant without mxaF or the region from the integrated plasmid was successfully created.

\section{Cell Growth and Methanol Accumulation in the OB3b $\Delta m \times a F$ Mutant}

To evaluate our strategic design for methanol production and cell growth, we examined the OB3b $\Delta m x a F$ mutant construct in media with and without copper and cerium ions. We isolated the OB3b $\triangle m x a F$ mutant using selective growth media and 
A

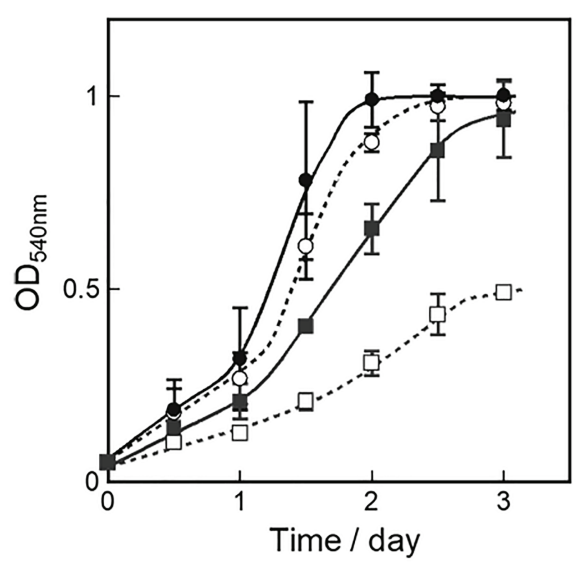

B

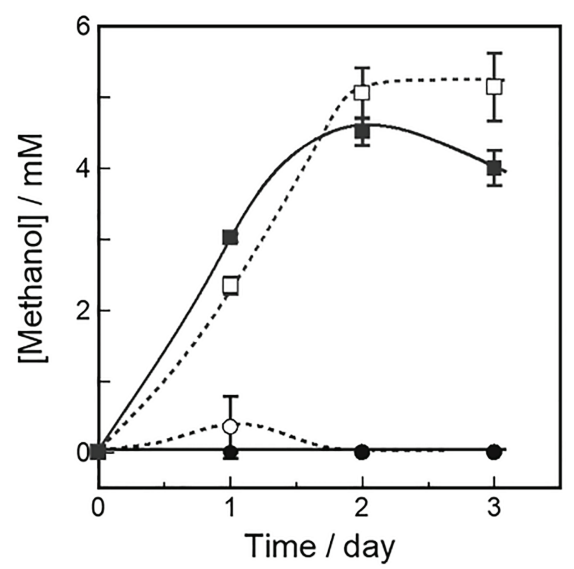

FIGURE 3 | Cell growth (A) and methanol production (B) in OB3b $\triangle m x a F$ mutant in presence of various copper and cerium concentrations. A starter culture grown in NMS with $25 \mu \mathrm{M}$ cerium ion was used as an inoculum to examine the growth and methanol production of the OB3b $\Delta m \times a F$ mutant in the fresh medium of four different culture conditions. $\bigcirc, 0 \mu \mathrm{M}$ copper ion plus $0 \mu \mathrm{M}$ cerium ion; $\boldsymbol{\bullet}, 0 \mu \mathrm{M}$ copper ion plus $25 \mu \mathrm{M}$ cerium ion; $\square$, $10 \mu \mathrm{M}$ copper ion plus $0 \mu \mathrm{M}$ cerium ion; $\mathbf{\square}$ $10 \mu \mathrm{M}$ copper ion plus $25 \mu \mathrm{M}$ cerium ion. Errors bars: duplicate sample ranges.

incubated it in $20 \mathrm{ml}$ NMS medium containing $25 \mu \mathrm{M}$ cerium ion plus $0 \mu \mathrm{M}$ copper ion. After $\mathrm{OD}_{540}=0.8$ was attained, the culture was washed twice with $8 \mathrm{ml}$ fresh cerium- and copper-free NMS and transferred at a 1:9 ratio to NMS containing various copper and/or cerium ion concentrations. Figure $\mathbf{3 A}$ shows that the OB3b $\triangle m x a F$ mutant grew under all conditions but most slowly in the presence of $10 \mu \mathrm{M}$ copper ion and the absence of cerium ion. According to the previous report, this growth of the OB3b $\triangle m x a F$ mutant can be caused by any remaining $\mathrm{XoxF}$ and cerium ion in the initial seed cells (Farhan Ul Haque et al., 2016). To evaluate whether the OB3b $\triangle m x a F$ mutants could be propagated, the cultures were inoculated into identical fresh media under the same conditions. When the mutants were transferred to identical fresh media at initial $\mathrm{OD}_{540}=0.1$ (transfer $\left.\mathrm{A}\right)$, they grew in the presence of cerium ion (Supplementary Figure S3). However, the growth of the mutants was almost stopped in the absence of cerium ion. Therefore, this mutant required cerium ions to grow normally.

Methanol concentrations in the medium were measured simultaneously to evaluate methanol accumulation by the OB3b $\Delta m x a F$ mutant under each condition. Figure 3B shows that methanol accumulation was detected in all OB3b $\Delta m x a F$ mutants except those in the medium containing only $25 \mu \mathrm{M}$ cerium ion plus $0 \mu \mathrm{M}$ copper ion. Thus, methanol production with the OB3b $\triangle m x a F$ mutant can be realized by modulating the copper and cerium ion concentrations. Methanol production was also observed in the absence of an external electron donor. The methanol concentration was higher in the presence of $10 \mu \mathrm{M}$ copper ion than it was in its absence. When the OB3b $\triangle m x a F$ mutant was cultured in medium containing $10 \mu \mathrm{M}$ copper ion plus $0 \mu \mathrm{M}$ cerium ion, the maximum methanol content was $2.6 \mu \mathrm{mol} \cdot \mathrm{mg}^{-1} \mathrm{dry}$ cell weight $\cdot \mathrm{h}^{-1}$. One $\mathrm{OD}_{540}$ unit of $M$. trichosporium $\mathrm{OB} 3 \mathrm{~b}$ corresponds to $0.15 \mathrm{mg} \cdot \mathrm{ml}^{-1}$ dry cell weight. Within 2 days, the methanol was saturated in the medium containing $10 \mu \mathrm{M}$ copper ion plus $0 \mu \mathrm{M}$ cerium ion. The conversion efficiency of the accumulated methanol to the total amount of methane added to the reaction system was $\sim 0.3 \%$. The methanol accumulation in the OB3b $\triangle m x a F$ mutant decreased with increasing incubation time except in the presence of $10 \mu \mathrm{M}$ copper ion plus $0 \mu \mathrm{M}$ cerium ion. The recovery of methanol accumulation was assessed in the OB3b $\Delta m x a F$ mutant via inoculation experiments under two conditions. After the cultures were transferred to identical fresh medium at the initial $\mathrm{OD}_{540}=0.1$ (transfer A), no methanol production was observed under any conditions (Supplementary Figure S3B). When the cultures were transferred to the same volume of identical fresh medium (transfer B; constant cell density), the methanol accumulation was maintained in the presence of $10 \mu \mathrm{M}$ copper ion but stopped within 1 day after the medium was exchanged (Supplementary Figure S4). The methanol accumulation decreased on day 2 after the medium exchange in the presence of $10 \mu \mathrm{M}$ copper ion plus $25 \mu \mathrm{M}$ cerium ion. The cells were then replenished with identical fresh medium on day 5. Only slight methanol accumulation was detected in the medium containing $10 \mu \mathrm{M}$ copper ion plus $0 \mu \mathrm{M}$ cerium ion. Methanol accumulation was recovered by exchanging the medium but its activity nonetheless gradually declined thereafter. Though methanol accumulated in the presence of $25 \mu \mathrm{M}$ cerium, there was an evident reduction in methanol concentration in the presence of $10 \mu \mathrm{M}$ copper. Thus, XoxF consumed the methanol in the latter case.

We compared intracellular $\mathrm{MDH}$ expression by using $M$. trichosporium $\mathrm{OB} 3 \mathrm{~b}$ cultured in the medium under four different conditions. Western blotting of $\mathrm{MDH}$ showed that MxaF was absent in the OB3b $\triangle m x a F$ mutant under all conditions (Figure 4A). XoxF was expressed in the OB3b $\triangle m x a F$ mutant in the presence of cerium ion (Figure $4 \mathbf{B}$ ). XoxF expression was higher in the absence than the presence of copper ion and the same held true for the OB3b wild type. 
MxaF
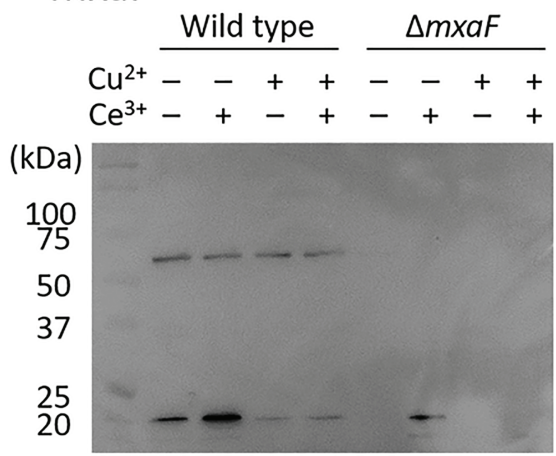

B

\section{XoxF}
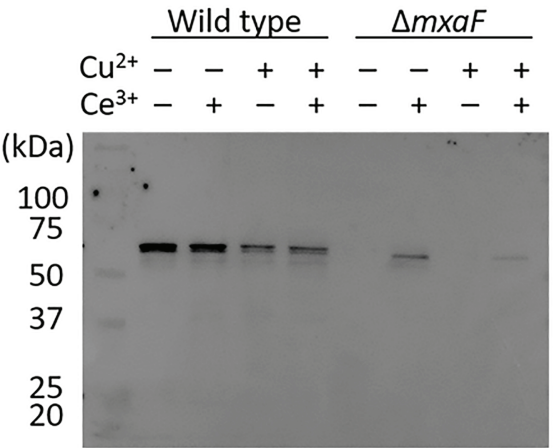

FIGURE 4 | Western blot of wild type and $\triangle m x a F$ mutant of $M$. trichosporium OB3b. Immunodetection of MxaF (A) and XoxF (B) using specific antisera. Molecular weight marker (in $\mathrm{kDa}$ ) of protein standards are shown on left.

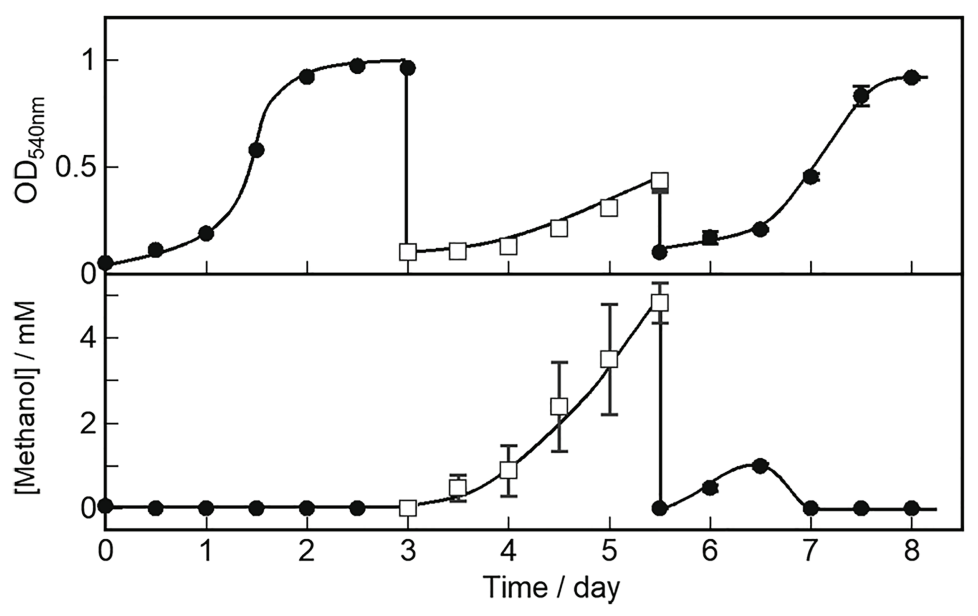

FIGURE 5 | OB3b $\triangle m x a F$ mutant switching between cell growth and methanol production conditions by modulating copper and cerium concentrations. $\bullet$, cell growth condition ( $0 \mu \mathrm{M}$ copper ion plus $25 \mu \mathrm{M}$ cerium ion); $\square$, methanol production condition (10 $\mu \mathrm{M}$ copper ion plus $0 \mu \mathrm{M}$ cerium ion). Errors bars: duplicate sample ranges.

\section{Switching Between Cell Growth and Methanol Production in OB3b $\Delta m x a F$ Mutant}

We characterized cell growth and methanol accumulation in the OB3b $\Delta m x a F$ mutant and demonstrated that its phenotype switched in response to changes in the metal ions in the medium. The cell growth curve and methanol concentration in the medium are shown in Figure 5. The OB3b $\triangle m x a F$ mutant was cultured with $0 \mu \mathrm{M}$ copper ion plus $25 \mu \mathrm{M}$ cerium ion. After 3 days, the cultures were collected, washed twice, and resuspended in NMS under conditions conducive to methanol production $(10 \mu \mathrm{M}$ copper ion plus $0 \mu \mathrm{M}$ cerium ion) to obtain $\mathrm{OD}_{540}=0.1$ after inoculation (first switch). After 2.5 days, the cultures were collected, washed twice, and resuspended in NMS under cell growth conditions to obtain the initial $\mathrm{OD}_{540}=0.1$ (second switch). In the first switch, the growth rate of the OB3b $\Delta m x a F$ mutant decreased and methanol accumulation increased without the addition of any external electron source. The amount of methanol per unit cell (mg dry cell weight) increased until 1.5 days and then remained constant until 2.5 days (Supplementary Figure S5). In the second switch, the growth rate was restored to the same level as that of the first step. Methanol accumulation increased until day 1 but the methanol disappeared by day 1.5 when the cells entered the log phase. Therefore, cell growth was recovered because XoxF expression consumed methanol. The foregoing results disclosed that the OB3b $\Delta m x a F$ mutant alter its phenotype and XoxF MDH expression can be controlled by altering the medium such that it favors cell growth or methanol production.

\section{DISCUSSION}

The culture of whole-cell methanotrophs requires a strategic design to optimize both methanol production and cell growth. 
In the present study, we characterized the continuous cell growth and methanol production in the OB3b $\Delta m x a F$ mutant in nutrient media containing four different copper and cerium ion configurations (Table 3). We modulated the medium conditions to promote cell growth $(0 \mu \mathrm{M}$ copper ion plus $25 \mu \mathrm{M}$ cerium ion) and methanol production (10 $\mu \mathrm{M}$ copper ion plus $0 \mu \mathrm{M}$ cerium ion) and switch the OB3b $\Delta m x a F$ mutant phenotype. The mutant presented with nearly the same growth as the wild type under conditions conducive to cell growth and it expressed XoxF in the presence of cerium ion. Figure 3A shows that mutant grew under all conditions but had a longer lag phase in the presence than the absence of copper ion. Furthermore, its overall growth was slowest under conditions conducive to methanol production. For transfer A (Supplementary Figure S3), the OB3b $\triangle m x a F$ mutant grew best under cell growth conditions. Though it also grew in the presence of $10 \mu \mathrm{M}$ copper and $25 \mu \mathrm{M}$ cerium, its lag phase there was longer than it was under cell growth conditions. Cell growth was strongly inhibited when XoxF was not expressed and cerium ion was absent (Figure 4B). Mutant growth under each condition of the present study resembled that of another M. trichosporium OB3b mxaF knockout mutant reported by Muhammad Farhan Ul Haque et al. (2016). These authors also indicated a reduction in cell growth in the presence of copper ions and total cell growth arrest in the absence of cerium ions. Thus, the OB3b $\triangle m x a F$ mutant can grow using any remaining XoxF and cerium ion in the initial seed cells. It was shown that $>98 \%$ of the added cerium is associated with microbial biomass (Farhan Ul Haque et al., 2015).

Methanol accumulation by the OB3b $\triangle m x a F$ mutant occurred in the medium containing copper ion. This condition is a requirement for pMMO expression (Figure 3B). Methane consumption was higher in whole-cell $M$. trichosporium OB3b expressing sMMO than it was in whole-cell $M$. trichosporium OB3b expressing pMMO (Sirajuddin and Rosenzweig, 2015). However, methanol did not accumulate in this mutant in the absence of copper and cerium ions which causes sMMO expression to predominate and represses XoxF (Figure 3B). Hence, methanol from sMMO was consumed more efficiently than methanol from $\mathrm{pMMO}$ by the remaining XoxF. The foregoing results and a previous study showed that pMMO forms a supercomplex with MxaF MDH (Culpepper and Rosenzweig, 2014). Coupling between pMMO and XoxF MDH

TABLE 3 | Characterization of the continuous cell growth and methanol production in the OB3b $\triangle m x a F$ mutant under four conditions containing copper and cerium ions.

\begin{tabular}{|c|c|c|c|c|}
\hline $\begin{array}{l}\text { Metal ions in } \\
\text { medium }\end{array}$ & $\begin{array}{l}\text { MMO } \\
\text { type }\end{array}$ & MDH type & $\begin{array}{l}\text { Continuous } \\
\text { cell growth }\end{array}$ & $\begin{array}{l}\text { Methanol } \\
\text { production }\end{array}$ \\
\hline $\begin{array}{l}0 \mu \mathrm{M} \mathrm{Cu}^{2+} \text { plus } \\
0 \mu \mathrm{M} \mathrm{Ce}^{3+}\end{array}$ & sMMO & - & - & - \\
\hline $\begin{array}{l}0 \mu \mathrm{MCu}^{2+} \text { plus } \\
25 \mu \mathrm{MCe}^{3+}\end{array}$ & sMMO & XoxF & ++ & - \\
\hline $\begin{array}{l}10 \mu \mathrm{MCu}^{2+} \text { plus } \\
0 \mu \mathrm{MCe}^{3+}\end{array}$ & pMMO & - & - & ++ \\
\hline $\begin{array}{l}10 \mu \mathrm{MCu}^{2+} \text { plus } \\
25 \mu \mathrm{MCe}^{3+}\end{array}$ & pMMO & XoxF & + & + \\
\hline
\end{tabular}

is weaker than coupling between $\mathrm{pMMO}$ and $\mathrm{MxaF} \mathrm{MDH}$. Methanol may accumulate in cells expressing XoxF and pMMO because of weak coupling between XoxF and pMMO. XoxF does not oxidize methanol from pMMO as effectively as MxaF. This hypothesis explains the results shown in Figure 3B and Supplementary Figure S4. In the presence of $10 \mu \mathrm{M}$ copper ion plus $25 \mu \mathrm{M}$ cerium ion, the aforementioned mutant could accumulate methanol as it does under methanol production conditions. In the former scenario, however, it consumes any methanol generated thereafter. Despite pMMO and XoxF expression in the presence of $10 \mu \mathrm{M}$ copper ion plus $25 \mu \mathrm{M}$ cerium ion (Farhan Ul Haque et al., 2015, 2016), there was no methanol accumulation in the case of transfer A (Supplementary Figure S3). Methane oxidation by pMMO is evident in the presence of $10 \mu \mathrm{M}$ copper ion plus $25 \mu \mathrm{M}$ cerium ion. Hence, the mutant may regulate the balance between $\mathrm{pMMO}$ and $\mathrm{XoxF}$ and changes to the state appropriate for methane utilization. The methanol concentration reached saturation $(\sim 6 \mathrm{mM})$ in the OB3b $\triangle m x a F$ mutant grown in medium containing $10 \mu \mathrm{M}$ copper ion plus $0 \mu \mathrm{M}$ cerium ion (Figure 3B). Methanol accumulation was arrested as methanol inhibited pMMO activity reversibly (Furuto et al., 1999). The observed recovery of methanol accumulation in the OB3b $\triangle m x a F$ mutant after the fresh medium was exchanged corroborated this hypothesis (Supplementary Figure S4). One way to overcome this limitation is to use a continuous culture system wherein the medium is exchanged constantly and the methanol is removed.

To the best of our knowledge, this study is the first to report methanol production using a genetically modified methanotroph lacking MDH activity. Changing the phenotype of this mutant by altering the metal ion concentrations in its growth medium facilitates the recovery of bacterial enzymes, reducing equivalents, and methanol production. Therefore, this mutant can function as a methanol production biocatalyst for longer periods of time than conventional methods. A previous study on methanol production by methanotrophs revealed that several MDH inhibitors have been implemented to stop methanol metabolism. Furuto et al. (1999) reported semicontinuous methanol synthesis with $M$. trichosporium $\mathrm{OB} 3 \mathrm{~b}$ in the presence of the MDH inhibitor cyclopropanol. The methanol production rate was $3.2 \mu \mathrm{mol} \cdot \mathrm{mg}^{-1}$ cell. $\mathrm{h}^{-1}$ after the addition of $14.3 \mathrm{mM}$ sodium formate. Methanol production rates of $6.0 \mu \mathrm{mol} \cdot \mathrm{mg}^{-1}$ cell. $\mathrm{h}^{-1}$ (Mehta et al., 1987) and $2.6 \mu \mathrm{mol} \cdot \mathrm{mg}^{-1}$ cell. $\mathrm{h}^{-1}$ (In Yeub et al., 2015) were reported for M. trichosporium OB3b. The methanol production rate for $M$. trichosporium OB3b mutant in this study $\left(2.6 \mu \mathrm{mol} \cdot \mathrm{mg}^{-1}\right.$ cell. $\left.\mathrm{h}^{-1}\right)$ was similar to those of previous reports. The mutant had the same methanol production capacity as the wild type subjected to $\mathrm{MDH}$ inhibitors. However, the OB3b $\triangle m x a F$ mutant requires time to switch between cell growth and methanol production. After its phenotype was switched by changing the growth medium, the former characteristics of the mutant were restored for $\sim 1$ day before the phenotype transitions occurred (Figure 5). This lag time may be explained by the persistence of XoxF $\mathrm{MDH}$ and/or pMMO from the previous state in the medium. One day after modulating the metal ion composition of the medium, the 
characteristics of the alternate phenotype may be observed in most cells under the new condition. Therefore, strategies are required to improve the efficacy of the switching system to reduce the lag time in the phenotypic change. In addition, the low methanol conversion efficiency for $M$. trichosporium OB3b mutant in this study $(\sim 0.3 \%$ of the total amount of methane added to the reaction system) is an issue that needs to be improved. In recent years, new bioreactors that are effective for methanotrophs have been reported (Chen et al., 2020; Valverde-Pérez et al., 2020). It is expected that more efficient carbon conversion can be achieved by using these bioreactors.

Here, the $M$. trichosporium OB3b mutant of mxaF knockout (OB3b $\Delta m x a F$ mutant) was constructed by a double homologous recombination method and $s a c B$ was used as a counterselectable marker. The OB3b $\Delta m x a F$ mutant normally grows by expressing $\mathrm{XoxF} \mathrm{MDH}$ in media containing cerium ion. It accumulates methanol by expressing pMMO in media containing copper ion and no external metabolic electron donors. The phenotype of the OB3b $\Delta m x a F$ mutant can be altered between that which is found under cell growth conditions and that which occurs under methanol production conditions by changing the cerium and copper ion content in the growth media. Phenotype switching in the OB3b $\triangle m x a F$ mutant achieves continuous methanol production by restoring reducing power. The present study proposes an innovative strategy for methanol production using genetically engineered, metabolically modified methanotrophs. Although it is not yet cost-effective for industrial methanol production, this study shows a new possibility for methanol production using methanotrophs. Subsequent research should focus on optimizing the medium conditions and culture system under which the mutant could serve as an industrial biocatalyst converting methane to methanol.

\section{DATA AVAILABILITY STATEMENT}

The raw data supporting the conclusions of this article will be made available by the authors, without undue reservation.

\section{AUTHOR CONTRIBUTIONS}

$\mathrm{HI}$ and $\mathrm{KY}$ conducted the experiments. MI and $\mathrm{KH}$ assisted in the experiment design and gave a hand in performing the experiments. HI and TK analyzed the data and wrote the manuscript. All authors contributed to the article and approved the submitted version.

\section{REFERENCES}

Bjorck, C. E., Dobson, P. D., and Pandhal, J. (2018). Biotechnological conversion of methaneto methanol: evaluation of progress and potential. AIMS Bioeng. 5, 1-38. doi: 10.3934/bioeng.2018.1.1

Chen, Y. -Y., Ishikawa, M., Suzuki, R., Ito, H., Kamachi, T., and Hori, K. (2020). Evaluation of methane degradation performance in microbial gasphase reactions using effectively immobilized methanotrophs. Biochem. Eng. J. 154:107441. doi: 10.1016/j.bej.2019.107441

\section{FUNDING}

This study was conducted under the JPMJAL1402 Project of the Advanced Low Carbon Technology Research and Development Program (ALCA) funded by the Japan Science and Technology Agency (JST).

\section{ACKNOWLEDGMENTS}

We would like to thank Editage (www.editage.com) for English language editing.

\section{SUPPLEMENTARY MATERIAL}

The Supplementary Material for this article can be found online at: https://www.frontiersin.org/articles/10.3389/fmicb.2021.639266/ full\#supplementary-material

Supplementary Figure S1 | Plasmid construction protocol. (A) pJQ200SK UpDw, (B) pK18mobsacB_UpDw. Suicide plasmids used $\mathrm{Gm}^{r}$ (gentamicin resistance gene), $\mathrm{Km}^{r}$ (kanamycin resistance gene), and sacB as counterselectable markers.

Supplementary Figure S2 I PCR analysis of single crossover strains in genomic DNA of $M$. trichosporium OB3b::pK18mobsacB_UpDw using primer sets $A$ ( $m \times a F \_u p s t-F 2$ and mxaF_mid-R) and B (mxaF_dwst-R2 and mxaF_ mid-F). (A) Outline of experiment. (B) Electrophoresis of colony PCR amplicon using primer sets A and B. M, marker; WT, wild type. Red square: PCR product of recombination between downstream regions. Blue square: PCR product of recombination between upstream regions.

Supplementary Figure S3 | Cell growth and methanol accumulation in OB3b $\Delta m x a F$ mutant after subculture with identical media at initial $\mathrm{OD}_{540}=0.1 .0$, $0 \mu \mathrm{M}$ copper ion plus $0 \mu \mathrm{M}$ cerium ion; $\bullet, 0 \mu \mathrm{M}$ copper ion plus $25 \mu \mathrm{M}$ cerium ion; $\square, 10 \mu \mathrm{M}$ copper ion plus $0 \mu \mathrm{M}$ cerium ion; $\square, 10 \mu \mathrm{M}$ copper ion plus $25 \mu \mathrm{M}$ cerium ion.

Supplementary Figure S4 | Continuous methanol production using OB3b $\Delta m \times a F$ mutant in presence of various copper and cerium concentrations. $\bigcirc$, $0 \mu \mathrm{M}$ copper ion plus $0 \mu \mathrm{M}$ cerium ion; $0,0 \mathrm{M}$ copper ion plus $25 \mu \mathrm{M}$ cerium ion; $\square, 10 \mu \mathrm{M}$ copper ion plus $0 \mu \mathrm{M}$ cerium ion; $\square, 10 \mu \mathrm{M}$ copper ion plus $25 \mu \mathrm{M}$ cerium ion. Exchange of identical fresh media containing all cultures on days 3 and 5.

Supplementary Figure S5 | OB3b $\Delta m x a F$ mutant switching between cell growth and methanol production conditions by modulating copper and cerium concentrations. One $\mathrm{OD}_{540}$ unit of $M$. trichosporium OB3b corresponds to 0.15 $\mathrm{mg} \cdot \mathrm{ml}^{-1}$ dry cell weight. $\bullet$, cell growth condition ( $0 \mu \mathrm{M}$ copper ion plus $25 \mu \mathrm{M}$ cerium ion); $\square$, methanol production condition (10 $\mu \mathrm{M}$ copper ion plus $0 \mu \mathrm{M}$ cerium ion). Errors bars: duplicate sample ranges.

Conrad, R. (2009). The global methane cycle: recent advances in understanding the microbial processes involved. Environ. Microbiol. Rep. 1, 285-292. doi: 10.1111/j.1758-2229.2009.00038.x

Cox, J. M., Day, D. J., and Anthony, C. (1992). The interaction of methanol dehydrogenase and its electron acceptor, cytochrome $\mathrm{cL}$ in methylotrophic bacteria Biochim. Biophys. Act 1119, 97-106. doi: 10.1016/0167-4838(92)90240-E

Culpepper, M. A., and Rosenzweig, A. C. (2014). Structure and protein-protein interactions of methanol dehydrogenase from Methylococcus capsulatus (Bath). Biochemistry 53, 6211-6219. doi: 10.1021/bi500850j 
Dales, S. L., and Anthony, C. (1995). The interaction of methanol dehydrogenase and its cytochrome electron acceptor. Biochem. J. 312, 261-265. doi: 10.1042/ bj3120261

Duan, C., Luo, M., and Xing, X. (2011). High-rate conversion of methane to methanol by Methylosinus trichosporium OB3b. Bioresour. Technol. 102, 7349-7353. doi: 10.1016/j.biortech.2011.04.096

Farhan Ul Haque, M., Gu, W., DiSpirito, A. A., and Semrau, J. D. (2016). Marker exchange mutagenesis of mxaF, encoding the large subunit of the Mxa methanol dehydrogenase, in Methylosinus trichosporium OB3b. Appl. Environ. Microbiol. 82, 1549-1555. doi: 10.1128/aem.03615-15

Farhan Ul Haque, M., Kalidass, B., Bandow, N., Turpin, E. A., DiSpirito, A. A., and Semrau, J. D. (2015). Cerium regulates expression of alternative methanol dehydrogenases in Methylosinus trichosporium OB3b. Appl. Environ. Microbiol. 81, 7546-7552. doi: 10.1128/aem.02542-15

Frank, J. Jr., van Krimpen, S. H., Verwiel, P. E. J., Jongejan, J. A., Mulder, A. C., and Duine, J. A. (1989). On the mechanism of inhibition of methanol dehydrogenase by cyclopropane-derived inhibitors. Eur. J. Biochem. 184, 187-195. doi: 10.1111/j.1432-1033.1989.tb15006.x

Furuto, T., Takeguchi, M., and Okura, I. (1999). Semicontinuous methanol biosynthesis by Methylosinus trichosporium OB3b. J. Mol. Catal. A Chem. 144, 257-261. doi: 10.1016/S1381-1169(99)00007-2

Groom, J. D., Ford, S. M., Pesesky, M. W., and Lidstrom, M. E. (2019). A mutagenic screen identifies a tonb-dependent receptor required for the lanthanide metal switch in the type i methanotroph "Methylotuvimicrobium buryatense" 5GB1C. J. Bacteriol. 201:e00120-19. doi: 10.1128/JB.00120-19

Han, J. -S., Ahn, C. -M., Mahanty, B., and Kim, C. -G. (2013). Partial oxidative conversion of methane to methanol through selective inhibition of methanol dehydrogenase in methanotrophic consortium from landfill cover soil. Appl. Biochem. Biotechnol. 171, 1487-1499. doi: 10.1007/s12010-013-0410-0

Hibi, Y., Asai, K., Arafuka, H., Hamajima, M., Iwama, T., and Kawai, K. (2011). Molecular structure of $\mathrm{La}^{3+}$-induced methanol dehydrogenase-like protein in Methylobacterium radiotolerans. J. Biosci. Bioeng. 111, 547-549. doi: 10.1016/j.jbiosc.2010.12.017

Hwang, I. Y., Hur, D. H., Lee, J. H., Park, C. -H.., Chang, I. S., and Lee, J. W., et al. (2015). Batch conversion of methane to methanol using Methylosinus trichosporium OB3b as biocatalyst. J. Microbiol. Biotechnol. 25, 375-380. doi: 10.4014/jmb.1412.12007

Keltjens, J. T., Pol, A., Reimann, J., and Op den Camp, H. J. M. (2014). PQQdependent methanol dehydrogenases: rare-earth elements make a difference. Appl. Microbiol. Biotechnol. 98, 6163-6183. doi: 10.1007/s00253-014-5766-8

Kim, H. G., Han, G. H., and Kim, S. W. (2010). Optimization of lab scale methanol production by Methylosinus trichosporium OB3b. Biotechnol. Bioprocess Eng. 15, 476-480. doi: 10.1007/s12257-010-0039-6

Lee, S. G., Goo, J. H., Kim, H. G., Oh, J. -I., Kim, Y. M., and Kim, S. W. (2004). Optimization of methanol biosynthesis from methane using Methylosinus trichosporium OB3b. Biotechnol. Lett. 26, 947-950. doi: 10.1023/B:bile.00000 25908.19252.63

Martin, H., and Murrell, J. C. (1995). Methane monooxygenase mutants of Methylosinus trichosporium constructed by marker-exchange mutagenesis. FEMS Microbiol. FEMS Microbiol. Lett. 127, 243-248. doi: 10.1111/j.1574-6968.1995.tb07480.x

Mehta, P. K., Ghose, T. K., and Mishra, S. (1991). Methanol biosynthesis by covalently immobilized cells of Methylosinus trichosporium: batch and continuous studies. Biotechnol. Bioeng. 37, 551-556. doi: 10.1002/bit.260370609

Mehta, P. K., Mishra, S., and Ghose, T. K. (1987). Methanol accumulation by resting cells of Methylosinus trichosporium (I). J. Gen. Appl. Microbiol. 33, 221-229. doi: 10.2323/jgam.33.221

Nakagawa, T., Mitsui, R., Tani, A., Sasa, K., Tashiro, S., Iwama, T., et al. (2012). A catalytic role of XoxF1 as $\mathrm{La}^{3+}$-dependent methanol dehydrogenase in Methylobacterium extorquens strain AM1. PLoS One 7:e50480. doi: 10.1371/ journal.pone.0050480

Pen, N., Soussan, L., Belleville, M. P., Sanchez, J., Charmette, C., and Paolucci-Jeanjean, D. (2014). An innovative membrane bioreactor for methane biohydroxylation. Bioresour. Technol. 174, 42-52. doi: 10.1016/j.biortech.2014. 10.001

Pen, N., Soussan, L., Belleville, M. P., Sanchez, J., and Paolucci-Jeanjean, D. (2016). Methane hydroxylation by Methylosinus trichosporium OB3b: monitoring the biocatalyst activity for methanol production optimization in an innovative membrane bioreactor. Biotechnol. Bioprocess Eng. 21, 283-293. doi: 10.1007/ s12257-015-0762-0
Pol, A., Barends, T. R. M., Dietl, A., Khadem, A. F., Eygensteyn, J., Jetten, M. S. M., et al. (2014). Rare earth metals are essential for methanotrophic life in volcanic mudpots. Environ. Microbiol. 16, 255-264. doi: 10.1111/1462-2 920.12249

Quandt, J., and Hynes, M. F. (1993). Versatile suicide vectors which allow direct selection for gene replacement in gram-negative bacteria. Gene 127, 15-21. doi: 10.1016/0378-1119(93)90611-6

Sambrook, J., and Russell, D. W. (2006). The inoue method for preparation and transformation of competent E. coli: "ultra-competent" cells. CSH Protoc. 2006:pdb.prot3944. doi: 10.1101/pdb.prot3944

Schäfer, A., Tauch, A., Jäger, W., Kalinowski, J., Thierbach, G., and Pühler, A. (1994). Small mobilizable multi-purpose cloning vectors derived from the Escherichia coli plasmids pK18 and pK19: selection of defined deletions in the chromosome of Corynebacterium glutamicum. Gene 145, 69-73. doi: 10.1016/0378-1119(94)90324-7

Semrau, J. D., Jagadevan, S., DiSpirito, A. A., Khalifa, A., Scanlan, J., Bergman, B. H., et al. (2013). Methanobactin and MmoD work in concert to act as the 'copper-switch' in methanotrophs. Environ. Microbiol. 15, 3077-3086. doi: 10.1111/1462-2920.12150

Shimoda, M., and Okura, I. (1990). Effect of cyclopropane treatment of Methylosinus trichosporium (OB3b) for methane hydroxylation. J. Chem. Soc. Chem. Comm. 7, 533-534. doi: 10.1039/C39900000533

Shindell, D., Kuylenstierna, J. C. I., Vignati, E., van Dingenen, R., Amann, M., Klimont, Z., et al. (2012). Simultaneously mitigating near-term climate change and improving human health and food security. Science 335, 183-189. doi: $10.1126 /$ science. 1210026

Simon, R. (1984). High-frequency mobilization of gram-negative bacterial replicons by the in vitro constructed Tn5-Mob transposon. Mol. Gen. Genet. 196, 413-420. doi: 10.1007/BF00436188

Sirajuddin, S., and Rosenzweig, A. C. (2015). Enzymatic oxidation of methane. Biochemistry 54, 2283-2294. doi: 10.1021/acs.biochem.5b00198

Takeguchi, M., Furuto, T., Sugimori, D., and Okura, I. (1997). Optimization of methanol biosynthesis by Methylosinus trichosporium OB3b: an approach to improve methanol accumulation. Appl. Biochem. Biotechnol. 68, $143-152$

U.S. Environmental Protection Agency (EPA) (2019). Global non- $\mathrm{CO}_{2}$ greenhouse gas emission projections \& mitigation: 2015-2050. Report No.: EPA 430R-19-010. Available at: https://www.epa.gov/sites/production/files/2019-09/ documents/epa_non-co2_greenhouse_gases_rpt-epa430r19010.pdf (Accessed Novenber 10, 2019).

Valverde-Pérez, B., Xing, W., Zachariae, A. A., Skadborg, M. M., Kjeldgaard, A. F., Palomo, A., et al. (2020). Cultivation of methanotrophic bacteria in a novel bubble-free membrane bioreactor for microbial protein production. Bioresour. Technol. 310:123388. doi: 10.1016/j.biortech.2020.123388

Vu, H. N., Subuyuj, G. A., Vijayakumar, S., Good, N. M., Martinez-Gomez, N. C., and Skovran, E. (2016). Lanthanide-dependent regulation of methanol oxidation systems in Methylobacterium extorquens AM1 and their contribution to methanol growth. J. Bacteriol. 198, 1250-1259. doi: 10.1128/jb.00937-15

Whittenbury, R., Phillips, K., and Wilkinson, J. (1970). Enrichment, isolation and some properties of methane-utilizing bacteria. Microbiology 61, 205-218. doi: 10.1099/00221287-61-2-205

Williams, P. A., Coates, L., Mohammed, F., Gill, R., Erskine, P. T., Coker, A., et al. (2005). The atomic resolution structure of methanol dehydrogenase from Methylobacterium extorquens. Acta Crystallogr. D Biol. Crystallogr. 61, 75-79. doi: 10.1107/S0907444904026964

Yoon, S., Semrau, J. D., and DiSpirito, A. A. (2010). Methanotrophs and copper. FEMS Microbiol. Rev. 34, 496-531. doi: 10.1111/j.1574-6976.2010.00212.x

Conflict of Interest: The authors declare that the research was conducted in the absence of any commercial or financial relationships that could be construed as a potential conflict of interest.

Copyright (C) 2021 Ito, Yoshimori, Ishikawa, Hori and Kamachi. This is an openaccess article distributed under the terms of the Creative Commons Attribution License (CC BY). The use, distribution or reproduction in other forums is permitted, provided the original author(s) and the copyright owner(s) are credited and that the original publication in this journal is cited, in accordance with accepted academic practice. No use, distribution or reproduction is permitted which does not comply with these terms. 